\title{
Thermally induced magnetic switching in bit-patterned media
}

Cite as: J. Appl. Phys. 122, 043907 (2017); https://doi.org/10.1063/1.4992808

Submitted: 17 March 2017 . Accepted: 26 June 2017 . Published Online: 31 July 2017

B. Pfau (D), C. M. Günther (D), T. Hauet (D), S. Eisebitt, and O. Hellwig
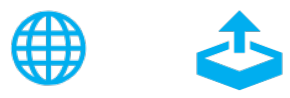

Export Citation

CrossMark

\section{ARTICLES YOU MAY BE INTERESTED IN}

Spin wave generation by surface acoustic waves

Journal of Applied Physics 122, 043904 (2017); https://doi.org/10.1063/1.4996102

Single shot ultrafast all optical magnetization switching of ferromagnetic Co/Pt multilayers Applied Physics Letters 111, 042401 (2017); https://doi.org/10.1063/1.4994802

Shapeable magnetoelectronics

Applied Physics Reviews 3, 011101 (2016); https://doi.org/10.1063/1.4938497

\section{Lock-in Amplifiers up to $600 \mathrm{MHz}$}
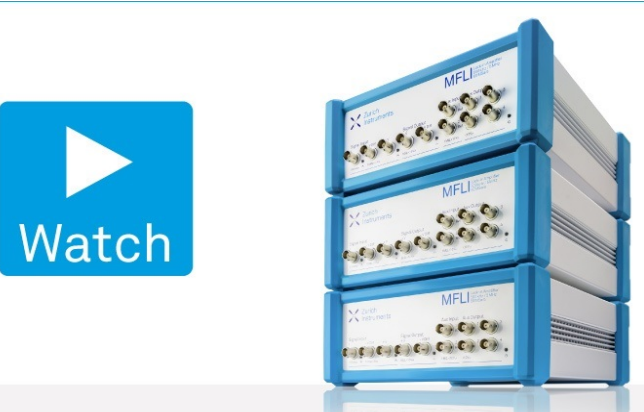


\title{
Thermally induced magnetic switching in bit-patterned media
}

\author{
B. Pfau, ${ }^{1, \text { a) }}$ C. M. Günther, ${ }^{2}$ T. Hauet, ${ }^{3,4}$ S. Eisebitt, ${ }^{1,2}$ and O. Hellwig ${ }^{3,5,6}$ \\ ${ }^{1}$ Max-Born-Institut, Max-Born-Str. 2A, 12489 Berlin, Germany \\ ${ }^{2}$ TU Berlin, Institut für Optik und Atomare Physik, Str. des 17. Juni 135, 10623 Berlin, Germany \\ ${ }^{3}$ San Jose Research Center, HGST a Western Digital Company, 3403 Yerba Buena Rd., San Jose, \\ California 95135, USA \\ ${ }^{4}$ Institut Jean Lamour, UMR 7198 CNRS-Université de Lorraine, Vandoeuvre-lès-Nancy, 54506, France \\ ${ }^{5}$ Helmholtz-Zentrum Dresden-Rossendorf, Institut für Ionenstrahlphysik und Materialforschung, \\ Bautzner Landstr. 400, 01328 Dresden, Germany \\ ${ }^{6}$ Institut für Physik, Technische Universität Chemnitz, 09126 Chemnitz, Germany
}

(Received 17 March 2017; accepted 26 June 2017; published online 31 July 2017)

\begin{abstract}
We have studied the thermal variation of the switching field of magnetic islands at room temperature. A model bit-pattern media composed of an assembly of islands with $80 \mathrm{~nm}$ width was fabricated by sputter deposition onto a pre-patterned substrate. Using direct magnetic-contrast imaging of the islands under applied field, we extract the switching probabilities of individual islands. Based on an analytical model for the thermally activated switching of the islands, we are able to determine the intrinsic magnetic anisotropy of each island and, consequentially, a distribution of anisotropies for the island ensemble investigated. In the distribution, we identify a separated group of islands with a particularly small anisotropy. We attribute this group to islands containing misaligned grains triggering the magnetic reversal. At room temperature and slow field sweep rates, the observed thermal broadening of the switching-field distribution is small compared to the intrinsic broadening. However, we illustrate that thermal fluctuations play a crucial role at high sweep rates by extrapolating our results to technological relevant regimes. Published by AIP Publishing.

[http://dx.doi.org/10.1063/1.4992808]
\end{abstract}

\section{INTRODUCTION}

Recording media comprising ordered arrays of lithographically patterned magnetic islands, so-called bit-patterned media (BPM), are one of the candidates to replace granular media in order to achieve recording densities well beyond $1 \mathrm{Tbit} / \mathrm{in}^{2}$ in the future. ${ }^{1}$ One of the main technological challenges in the development of these media is the reduction of the island-to-island variation of the field needed for magnetic reversal of the islands, which is expressed in the switching-field distribution (SFD). Primarily, the SFD is determined by two contributions, ${ }^{1,2}$ intrinsic variations among the islands and broadening due to dipolar fields from nearby islands. ${ }^{3,4}$ The intrinsic distribution of switching field has been particularly attributed to a distribution of intrinsic anisotropy. ${ }^{5}$ In turn, the origin of this anisotropy distribution must be related to structural variations among islands. It was proposed that misaligned grains may act as initial nucleation sites for the magnetic reversal and, thus, the presence or absence of such a trigger grain determines the island's switching field. ${ }^{6,7}$

In addition to the above-mentioned contributions, thermal fluctuations may also broaden the SFD. As the thermal energy at room temperature is very small compared to the energy barrier of magnetic reversal in recording media, the influence of thermal fluctuations is typically neglected. However, under applied magnetic field-during writing a bit-the energy barrier decreases and the reversal is

\footnotetext{
a) Author to whom correspondence should be addressed: bastian.pfau@ mbi-berlin.de.
}

triggered by thermal fluctuations. Experimentally, thermal fluctuations then manifest in a variation of the switching field of each individual island when repeatedly addressed. This variation is, thus, here referred to as the switching probability distribution (SPD). ${ }^{8}$ If the island is subjected to considerable dipolar fields from neighboring islands, the SPD will be additionally broadened due to different magnetic configurations of the surrounding islands. ${ }^{4,8,9}$

Here, we investigate the SPD in BPM samples with a negligible small dipolar interaction between islands in order to directly compare thermal and intrinsic broadening of the SFD. Using in-field imaging of the islands' magnetic states, we directly show a thermal variation of the switching field at room temperature. Our experimental procedure allows extracting the islands' SPDs, which we use to determine the magnetic anisotropy individually for each island. We are, thus, able to correlate the intrinsic SFD with an experimentally obtained distribution of intrinsic anisotropies. ${ }^{5}$ Finally, we extrapolate our results to technologically relevant conditions with high reversal speeds, where the influence of thermal fluctuations significantly increases.

\section{EXPERIMENTAL DETAILS}

The BPM sample was prepared by sputter deposition of a perpendicular anisotropy $\mathrm{Co} / \mathrm{Pd}$ multilayer $(\mathrm{Ta}(15) / \mathrm{Pd}(30) /$ $[\mathrm{Co}(5.5) / \mathrm{Pd}(8)]_{24} / \mathrm{Pd}(11) \AA$ ) onto a $\mathrm{Si}_{3} \mathrm{~N}_{4}$ membrane substrate that was pre-patterned to periodic square arrays of cuboidal pillars with a lateral size of $(80 \times 80) \mathrm{nm}^{2}$ and a height of $40 \mathrm{~nm}$. The magnetic islands form on top of the pillars. ${ }^{10}$ Material deposited in the trenches between the islands 
remains magnetically active but is decoupled from the islands in terms of exchange interaction. Transmissionelectron micrographs of plan-view sections of 24 selected islands ${ }^{7}$ have revealed an island size (area) distribution with a mean of $6100 \mathrm{~nm}^{2}$ and a width of $7 \%$ (standard deviation). Due to the uniform deposition process, we expect that height deviations of the multilayer are negligible. Vibrating-sample magnetometer measurements of the magnetic layer prepared as a continuous film on a sister sample yielded a saturation magnetization of $M_{\mathrm{s}}=(690 \pm 10) \mathrm{emu} \mathrm{cm}^{-3}$ and a crystal field uniaxial anisotropy of $K_{\mathrm{u}}=(5.7 \pm 0.3) \times 10^{6} \mathrm{erg} \mathrm{cm}^{-3}$ both normalized to the total multilayer thickness. The uniaxial anisotropy was calculated from in-plane and out-of-plane magnetization loops as described in Ref. 11.

We have analyzed the SPD for two differently patterned media with $240 \mathrm{~nm}$ and $200 \mathrm{~nm}$ pitch. For media with smaller pitch, we found that the SPD of the islands is additionally broadened by the strong dipole interactions between the islands ${ }^{4}$ due to a magnetic configuration that changes from run to run. ${ }^{8}$ Each island ensemble was imaged using soft-Xray Fourier-transform holography under a magnetic field applied in the out-of-plane direction. ${ }^{12,13}$ In the imaging experiment, the field of view (FOV) for each pattern array is fixed to a circular area with a diameter of $1.5 \mu \mathrm{m}$ and contained $N=29(240 \mathrm{~nm}$ pitch) and $42(200 \mathrm{~nm}$ pitch) islands [Fig. 1]. To determine the full SFD of the island ensembles, we have recorded hysteresis loops of both ensembles by imaging the magnetic state of the islands in ten hysteresis cycles (always starting from saturation at $-7.1 \mathrm{kOe}$ ) at 22 different applied field $(H)$ steps. The normalized net magnetization of the ensemble is then determined as $M(H)=2 n(H) / N-1$, where $n(H)$ denotes the number of reversed islands [Fig. 1(a)]. A fit of the loops with an asymmetric error function ${ }^{2,7}$ results in a width (standard deviation/coercive field) of the SFD of $15 \%$ (19\%) for the ensemble with $240 \mathrm{~nm}(200 \mathrm{~nm})$ pitch. $^{7}$ The SFD of the ensembles as shown in Fig. 1(a) [and later in Fig. 4(a)] is numerically calculated as the probability distribution $\mathrm{d} M(H) /(2 \mathrm{~d} H)$, where the factor $1 / 2$ accounts for the range $[-1: 1]$ of the normalized magnetization. Further details about the sample preparation and the imaging experiment can be found in our previous publications. ${ }^{4,7}$

In the conventional Arrhenius formulation, the life time of the magnetic state of a magnetic island is given by ${ }^{14}$

$$
\tau=\tau_{0} \exp \left[\frac{E_{\mathrm{b}}(H)}{k_{\mathrm{B}} T}\right] .
$$

The attempt frequency is estimated with $1 / \tau_{0}=10^{9} \mathrm{~Hz}$ and $k_{\mathrm{B}}$ is the Boltzmann constant. In our experiment, the temperature $T$ was equal to room temperature $((296 \pm 3) \mathrm{K})$. The energy barrier $E_{\mathrm{b}}$ for the island's magnetic reversal depends on the effectively applied field $H$ that includes the applied field and external demagnetizing fields stemming from the magnetized trench material and the surrounding islands. ${ }^{4}$ It is assumed that these external demagnetizing fields do not change during the reversal of the selected island. $E_{\mathrm{b}}$ can be assessed by the simple analytical expression resulting from integrating the island's magnetic energy over the full reversal ${ }^{15}$
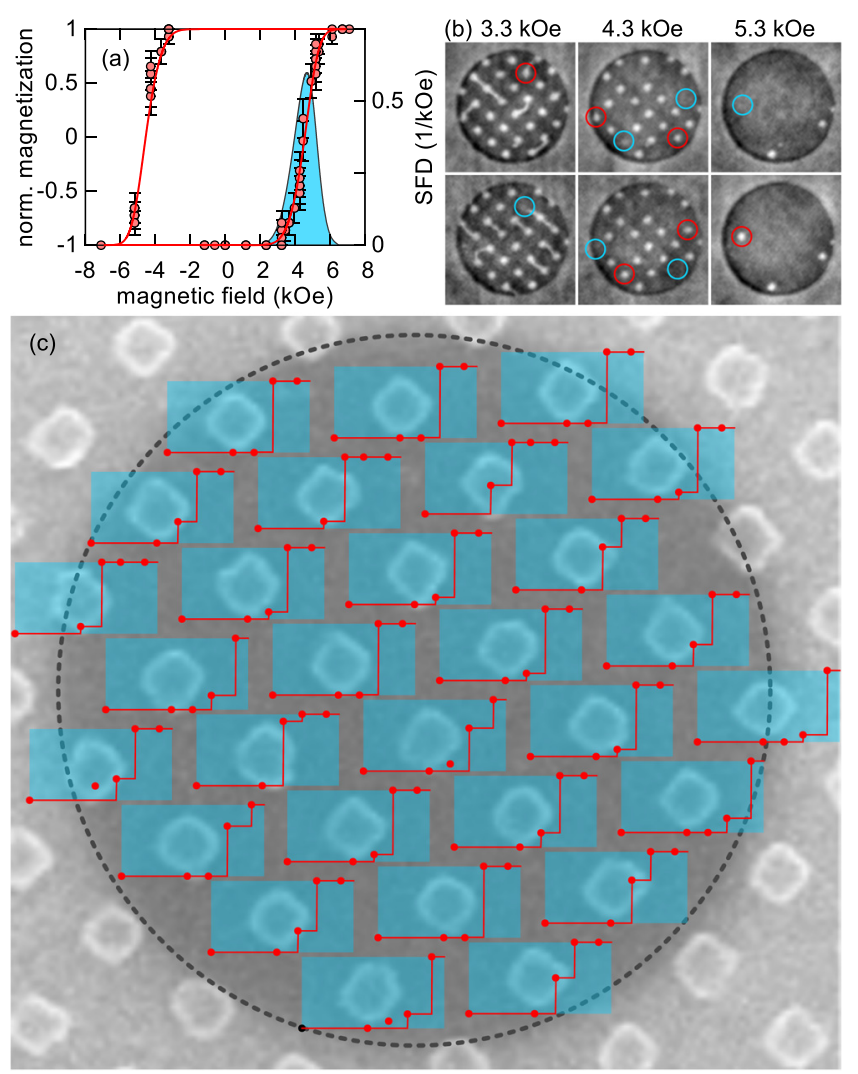

FIG. 1. (a) Magnetic hysteresis loop of the BPM array with $240 \mathrm{~nm}$ pitch. The data points show the net magnetization of the full imaged island ensemble extracted from FTH images (examples are shown in panel (b)) taken during ten independent hysteresis runs. The solid line is a fit to the data with an asymmetric error function where the SFD of the ensemble (filled curve) is derived from (cf. Ref. 4 for more details). (b) Selected images of the magnetic reversal of the same sample. Field steps are indicated on top. The field of view (FOV) is $1.5 \mu \mathrm{m}$ in diameter. The gray scale encodes the out-ofplane magnetization with the magnetization direction pointing into the plane for bright areas and out of the plane for dark areas. The images in the top row and in the bottom row were obtained in two different hysteresis runs. The colored circles point to differences in the switching of individual islands (red: island has switched; blue: island has not switched). (c) Individual reversal curves for each island (the scanning electron microscopy image in the background indicates the position of each island in the imaged FOV) compiled out of 10 independent switching events. The applied field axis (abscissa) in each plot scales from 0 to $7 \mathrm{kOe}$, and the reversal probability axis (ordinate) from 0 to 1 ( 0 means the island was never switched and 1 means that the island was reversed in 10 out of 10 runs). The dots indicate data points whereas the lines correspond to the fit assuming a thermally activated switching.

$$
E_{\mathrm{b}}(H)=K_{\mathrm{eff}} V\left(1-\frac{H M_{\mathrm{s}}}{2 K_{\mathrm{eff}}}\right)^{2}
$$

The effective anisotropy $K_{\text {eff }}$ is given by the uniaxial anisotropy $K_{\mathrm{u}}$ reduced by the shape anisotropy $M_{\mathrm{s}} H_{\text {intern }} / 2$ raised by the island's internal demagnetizing field $H_{\text {intern }}$. The magnitudes of all demagnetizing fields are taken from an analytical model as previously published in Ref. 4. From this model, we have calculated a shape anisotropy of $(1.95 \pm 0.10) \times 10^{6} \mathrm{erg} \mathrm{cm}^{-3}$ where the error accounts for a variation in the size of the islands. In magnetic islands being much larger than the critical length $\sqrt{A} / M_{\mathrm{s}} \approx 14 \mathrm{~nm}$ (exchange constant $A \approx 10^{-6} \mathrm{erg} \mathrm{cm}^{-1}$ ), the magnetization is not reversed by a quasi-coherent rotation in the whole island volume rather than by an incoherent rotation activated in a much smaller volume. ${ }^{6,16}$ Consequentially, the 
volume $V$ is identified with this activation volume. An incoherent rotation of the magnetization will also result in an energetically more favorable transition. Typically, this is accounted for by an exponent being smaller than 2 in (2). ${ }^{17,18}$ However, it is very difficult to experimentally distinguish different exponents ${ }^{17}$ and the approximation of a constant exponent of 2 is employed in this work.

From the life time given in (1), the probability that the magnetization of an island has not switched at a fixed field $H$ after a time $\Delta t$ is calculated as

$$
\omega(H)=\exp [-\Delta t / \tau(H)]
$$

When the applied field is swept from zero towards the anisotropy field $H_{\mathrm{a}}=2 K_{\mathrm{eff}} / M_{\mathrm{s}}$, the energy barrier will decrease and a switching event may occur due to thermal fluctuation before $H_{\mathrm{a}}$ is reached. In fact, in this scenario, both the field with the highest switching probability and the mean coercive field will always be smaller than the anisotropy field. The mathematical description of the thermally induced SFD observed in a repeated measurement of ramping the external field is based on the work of Kurkijärvi studying thermal fluctuations in Josephson junctions. ${ }^{19}$ Following this model, the SPD at a field $H$ is expressed as ${ }^{20,21}$

$p(H)=\frac{1}{v \tau_{0}} \exp \left(\frac{-E_{\mathrm{b}}(H)}{k_{\mathrm{B}} T}\right) \exp \left\{\frac{-1}{v \tau_{0}} \int_{0}^{H} \exp \left(\frac{-E_{\mathrm{b}}\left(H^{\prime}\right)}{k_{\mathrm{B}} T}\right) \mathrm{d} H^{\prime}\right\}$,

where $v$ denotes the fixed ramping rate of the external field $v=\mathrm{d} H / \mathrm{d} t$. Experimentally, (4) was successfully used to model the thermal SFD obtained from SQUID ${ }^{22}$ and anomalous Halleffect measurements ${ }^{17}$ of individual small magnetic particles. In contrast to these experiments where the field was swept at a constant rate, the field in the present imaging experiment was changed in large steps. In each step, the field was increased up to a certain value on the time scale of milliseconds and thenduring image acquisition-kept constant for approximately $10^{3} \mathrm{~s}$. In order to account for the discontinuous changes, we modified the model of Kurkijärvi following a consideration of Wang et $a l^{23}$ If $1-\omega(H)$ is the probability that the system overcomes the energy barrier at the magnetic field $H$, the probability that the island switches its magnetization between $H$ and $H+\mathrm{d} H$ will be the probability for passing the barrier times the probability that the switching has not yet occurred,

$$
p(H)=(1-\omega(H))\left[1-\int_{0}^{H} p\left(H^{\prime}\right) \mathrm{d} H^{\prime}\right] .
$$

While this consideration will again lead to (4) for a continuous sweep, the integral can be written as a series if the field is increased in a number of discrete steps $H_{j}$,

$$
p\left(H_{j+1}\right)=\left(1-\omega\left(H_{j+1}\right)\right)\left[1-\sum_{k=0}^{j} p\left(H_{k}\right)\right]
$$

where switching events in between the steps are neglected as the time for changing the field is much smaller than the following hold time.
In the present experiment, the switching probability of each individual island was determined at three different applied field magnitudes $(3.3 \mathrm{kOe}, 4.3 \mathrm{kOe}$, and $5.3 \mathrm{kOe})$ by imaging the ensembles in five independent full hysteresis runs always starting and returning from saturation at $\pm 7.1 \mathrm{kOe}$. Two example images for the island ensemble with $240 \mathrm{~nm}$ pitch at each field step are displayed in Fig. 1(b), clearly showing that a variation of switched island at a particular field exists. By taking images in both branches of the hysteresis, ten independent configurations for each field have been recorded in total. In addition, it is known from the hysteresis loop of the full island ensemble [Fig. 1(a)] that the island ensemble has full remanence (i.e., islands do not switch up to a field of at least $0 \mathrm{kOe}$ ) and that it saturates at $6.7 \mathrm{kOe}$. Both values were additionally used in the analysis.

The resulting set of field-dependent switching probabilities for each island was fitted applying the discrete model in (6). Three island-dependent parameters enter into the fit via (2): (i) $M_{\mathrm{s}}$ is treated as a constant under the assumption that the island's $M_{\mathrm{s}}$ does not deviate from the value obtained for the continuous film as the magnetization is mostly related to the total amount of material homogeneously deposited. ${ }^{24}$ (ii) $K_{\text {eff }}$ was individually determined for every island in the fit procedure because the island-to-island intrinsic variation of the switching field is predominately addressed to variations of the island's anisotropy. ${ }^{5}$ (iii) As the activation volume $V$ and the anisotropy are correlated in the fit, it was not possible to determine both properties for each island with significance. Instead, the fit was performed simultaneously for the whole island ensemble and a common volume for the ensemble was fitted. The assumption of an anisotropy distribution among the islands and a common activation volume is supported by the results of Ref. 5, but we cannot exclude that part of the fitted $K_{\text {eff }}$ distribution is related to an actual variation in $V$. As a test for the significance of the fit procedure, we fitted both island ensembles (with $240 \mathrm{~nm}$ and $200 \mathrm{~nm}$ pitch) separately.

\section{RESULTS AND DISCUSSION}

The experimentally obtained switching probabilities for each island in the first ensemble together with the fit of these probabilities using (6) are displayed in Fig. 1(c). The corresponding distribution of the islands' effective anisotropies for both ensembles can be found in Fig. 2. The distribution has a mean uniaxial anisotropy of $(3.4 \pm 0.3) \times 10^{6} \mathrm{erg} \mathrm{cm}^{-3}$ $\left((3.3 \pm 0.3) \times 10^{6} \mathrm{erg} \mathrm{cm}^{-3}\right)$ and a width (standard deviation) of $0.3 \times 10^{6} \mathrm{erg} \mathrm{cm}^{-3}\left(0.3 \times 10^{6} \mathrm{erg} \mathrm{cm}^{-3}\right)$ for the BPM ensemble with $240 \mathrm{~nm}(200 \mathrm{~nm})$ pitch. The activation volumes were fitted as $((9.1 \pm 0.6) \mathrm{nm})^{3}$ and $((9.2 \pm 0.6) \mathrm{nm})^{3}$, respectively. Both ensembles, thus, give equal results. The fitted activation volume is very similar to what was previously found for magnetic islands based on a $\mathrm{Co} / \mathrm{Pd}$ multilayer. ${ }^{5,25}$ The error bars as derived from the fit are mainly related to the high correlation between activation volume and anisotropy. Unfortunately, artifacts in the fitted distribution occur due to the chosen sampling of the magnetic field steps where images have been recorded. It turns out that the switching distribution of some islands could not be resolved 


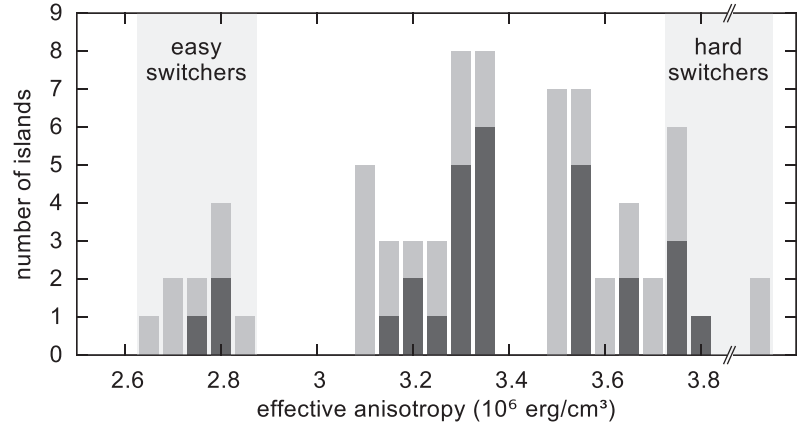

FIG. 2. Histogram of the anisotropy individually fitted for each island. The dark gray columns show the results for the BPM with $240 \mathrm{~nm}$ pitch while the light gray columns (put on top of the dark gray ones) correspond to the result from the BPM with $200 \mathrm{~nm}$ pitch.

as the field steps were too large [cf., e.g., the islands in the top row of Fig. 1(c)]. As a result, two gaps with missing islands in the $K_{\text {eff }}$ distribution around $2.95 \times 10^{6} \mathrm{erg} \mathrm{cm}^{-3}$ and $3.4 \times 10^{6} \mathrm{erg} \mathrm{cm}^{-3}$ appear [cf. Fig. 2]. Instead, the switching of those islands with an anisotropy located in these gaps is detected at the next higher field step and the fitted anisotropy is overestimated. The switching of the two hardest islands was never observed in the ten recorded hysteresis runs and these islands were excluded from the fit. From the fit of the other islands, it is evident that the effective anisotropy of these two islands is higher than $3.85 \times 10^{6} \mathrm{erg} \mathrm{cm}^{-3}$.

Despite the described limitations, the recorded images cover the switching statistics of the main part of the island ensembles and valuable information can be extracted from the anisotropy distribution found. Taking the shape anisotropy into account, the mean effective anisotropy $\left(3.35 \times 10^{6} \mathrm{erg}\right.$ $\mathrm{cm}^{-3}$ ) corresponds to an uniaxial anisotropy of approximately $K_{\mathrm{u}}=5.3 \times 10^{6} \mathrm{erg} \mathrm{cm}^{-3}$, which coincides (within the error bars) with the anisotropy found for the continuous film. The majority $(75 \%)$ of the islands has an effective anisotropy close to the mean value in a range between 3.15 and $3.75 \times 10^{6}$ erg $\mathrm{cm}^{-3}$ corresponding to a range width of $18 \%$ of the mean anisotropy. Nevertheless, the anisotropy of the magnetically softest and hardest islands diverges by more than $1.2 \times 10^{6} \mathrm{erg}$ $\mathrm{cm}^{-3}$ corresponding to approximately one third of the mean effective anisotropy.

In our previous study, ${ }^{7}$ we have investigated the structural properties of these selected islands that switch at particularly low (easy switchers) or high applied fields (hard switchers). We found that easy switchers contain misaligned grains that probably have a reduced anisotropy and, thus, trigger the island reversal. ${ }^{6}$ With the present analysis, we are now able to specifically attribute a magnetic anisotropy to these islands. In Fig. 3, we present high-resolution transmission electron micrographs of two islands (cf. Ref. 7 for experimental details) together with their anisotropy obtained in the fit as examples for easy and hard switchers. The darkfield image only highlights grains with the in-plane [ $\left[\begin{array}{lll}1 & 0 & 0\end{array}\right]$ orientations, i.e., grains that are significantly misaligned from the preferred face-centered cubic [ [ $\left.\begin{array}{lll}1 & 1 & 1\end{array}\right]$ out-of-plane crystal orientation. Since the crystal orientation is the second source of perpendicular anisotropy, which is highest for the [ $\left.\begin{array}{lll}1 & 1 & 1\end{array}\right]$ orientation, ${ }^{24}$ the selected regions will feature a
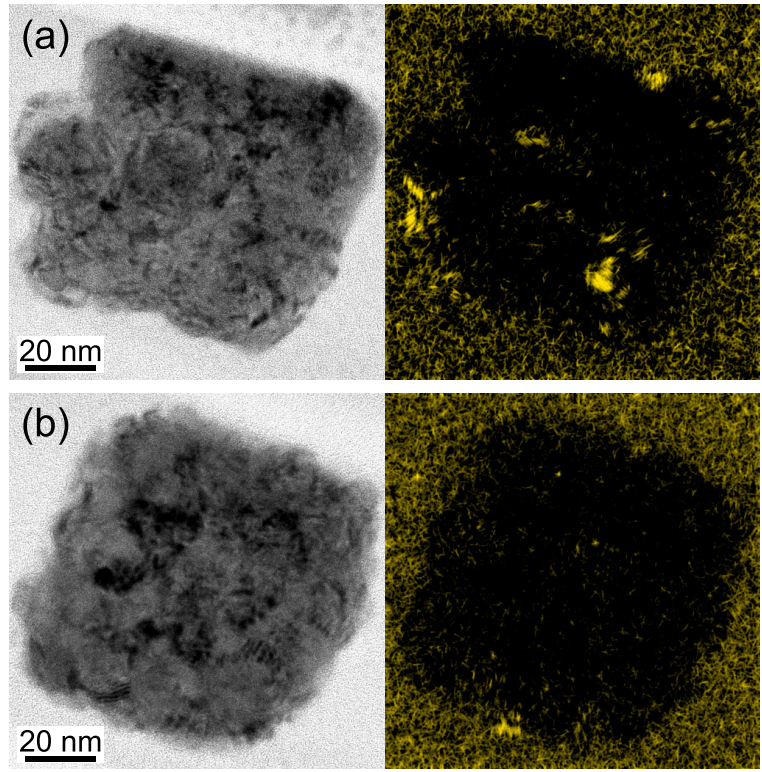

FIG. 3. Examples of high-resolution transmission electron micrographs of single islands: on the left hand side, bright-field images and, on the right hand side, dark-field images by selecting only (200) reflections (see Ref. 7 for experimental details). The dark field, thus, highlights grains with the inplane $\left[\begin{array}{lll}1 & 0 & 0\end{array}\right]$ orientations, i.e., grains that are significantly misaligned from the preferred face-centered cubic [ [ 1111$]$ out-of-plane crystal orientation. (a) "Easy switcher" containing misaligned grains. Fitted effective anisotropy: $(2.8 \pm 0.4) \times 10^{6} \mathrm{erg} \mathrm{cm}^{-3}$, (b) "Hard switcher" without misaligned grains. Fitted anisotropy: $(3.8 \pm 0.4) \times 10^{6} \mathrm{erg} \mathrm{cm}^{-3}$.

reduced anisotropy. However, it is to be noted that such a potential nucleus for the reversal is still exchange coupled with the other grains and its effective anisotropy is probably not the intrinsic value of the nucleus anisotropy alone.

In general, the histogram in Fig. 2 reveals that the anisotropies of the hard switchers are less deviating from the most frequent anisotropy than the easy switchers' anisotropies. While the hard islands are located in the upper tail of the main anisotropy distribution, the easy switchers form a separated group of islands with a significantly reduced anisotropy. This separated group of easy switchers is a contribution to the observed asymmetry of the intrinsic SFD [cf. Fig. 4(a)] in our experiments $^{4}$ and other studies on $\mathrm{Co} / \mathrm{Pd}$-based islands. ${ }^{2}$

In order to illustrate the impact of thermal effects on the SFD, we have calculated the SPD of an individual island based on the island's properties obtained in our analysis. For visualization in Fig. 4, we have employed (4) assuming a constant field sweep at a mean rate in the experiment on the order of $v=1 \mathrm{Oe} / \mathrm{s}$. We show the SPDs for the mean effective anisotropy as well as for the minimum and maximum anisotropies in the island ensemble with $240 \mathrm{~nm}$ pitch [Fig. 4(a)]. The width (standard deviation) of the SPDs at room temperature is about $0.13 \mathrm{kOe}$. The distributions have a pronounced asymmetry, which also contributes to the asymmetry of the total SFD. For comparison, we also show the full SFD of the ensemble as obtained from the hysteresis loop in Fig. 1(a). Due to the large inter-island distances in this bitpattern, the SFD width (standard deviation) of $0.67 \mathrm{kOe}$ is nearly entirely governed by the intrinsic variation of the switching fields. ${ }^{4}$ From the comparison, it is evident that thermal broadening only marginally contributes to the SFD 


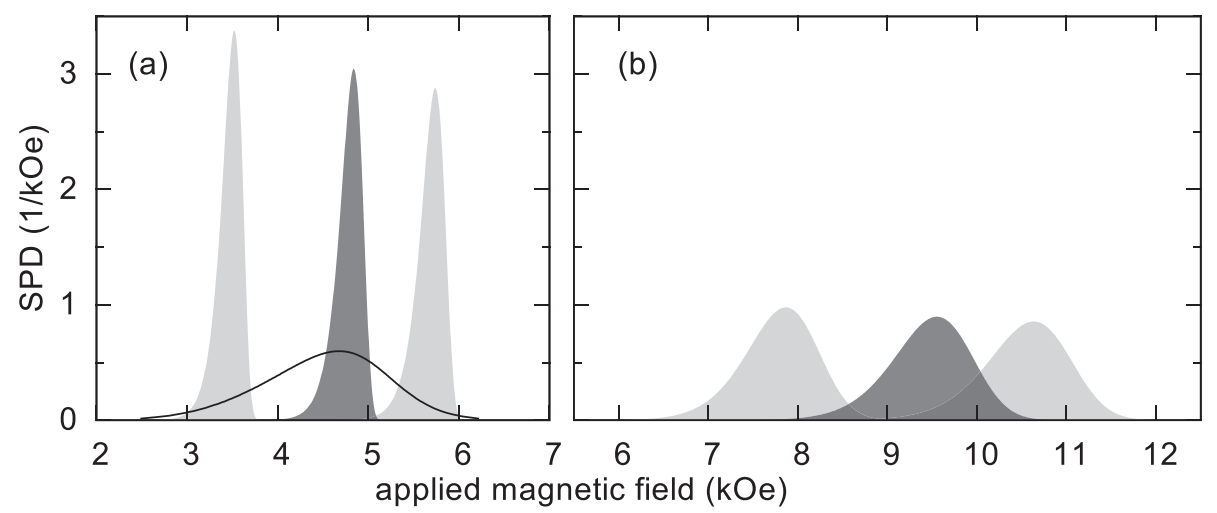

FIG. 4. Thermal single-island switching probability distributions assuming a continuous field sweep at a constant rate $v$ calculated using (4). (a) Situation in the imaging experiment with a mean sweep rate of $v \approx 1$ Oe/s. The dark gray filled curve shows the distribution for an island having the mean fitted anisotropy for the BPM with $240 \mathrm{~nm}$ pitch, and the light gray curves correspond to islands with minimum and maximum fitted anisotropy in the imaged ensemble. For comparison, the solid line indicates the SFD of the full ensemble as shown in Fig. 1(a). (b) The calculated switching distributions for the same island anisotropies as in (a) at a sweep rate of $v=10^{11} \mathrm{Oe} / \mathrm{s}$.

at room temperature and very low field-sweep velocity. However, this situation changes when increasing the velocity to a value relevant for the recording process in data-storage applications. Using the same model, we have calculated the SPDs for the same island anisotropies as before if the island was switched at a field rate of $10^{11} \mathrm{Oe} / \mathrm{s}$ [Fig. 4(b)]. Although this rate is still by at least one order of magnitude lower than realized in today's recording heads, this rate also marks the border of the validity of the Néel-Brown model being the basis for Eq. (4). ${ }^{26}$ Nevertheless, already at this rate, the mean switching field significantly shifts to higher values. In addition, the SPDs have broadened much stronger than the intrinsic SFD (which can be estimated from the switching field difference between the softest and the hardest island) and therefore thermal broadening significantly adds to the total SFD width. The same effects have been found in simulations for granular media. ${ }^{27}$

In summary, we have studied the thermal variation of the switching field of magnetic islands in a BPM context at room temperature. We have extracted the switching probabilities for individual islands from repeated imaging of two BPM ensembles in applied field. The SPDs were fitted with an analytical model yielding the intrinsic magnetic anisotropy for each island and an average activation volume. In the distribution of anisotropies, we have identified a separated group of islands with a particularly small anisotropy, which we attributed to islands containing misaligned grains triggering the magnetic reversal. We have shown that thermal broadening of the SFD is very small compared to the intrinsic variations at room temperature and slow field sweep rates. However, by extrapolating our results to technological relevant regimes, we illustrate that thermal fluctuations play a crucial role at high sweep rates.

\section{ACKNOWLEDGMENTS}

We thank $\mathrm{H}$. Yang for helping with the fabrication of the pre-patterned membrane substrates and T. Hennen for performing the vibrating-sample magnetometer characterization.

${ }^{1}$ T. R. Albrecht, H. Arora, V. Ayanoor-Vitikkate, J.-M. Beajour, D. Bedau, D. Berman, A. L. Bogdanov, Y.-A. Chapuis, J. Cushen, E. E. Dobisz, G.
Doerk, H. Gao, M. Grobis, B. Gurney, W. Hanson, O. Hellwig, T. Hirano, P.-O. Jubert, D. Kercher, J. Lille, Z. Liu, C. M. Mate, Y. Obukhov, K. C. Patel, K. Rubin, R. Ruiz, M. Schabes, L. Wan, D. Weller, T.-W. Wu, and E. Yang, "Bit Patterned Magnetic Recording: Theory, Media Fabrication, and Recording Performance," IEEE Trans. Magn. 51, 0800342 (2015). ${ }^{2}$ O. Hellwig, A. Berger, T. Thomson, E. Dobisz, Z. Z. Bandic, H. Yang, D. S. Kercher, and E. E. Fullerton, "Separating dipolar broadening from the intrinsic switching field distribution in perpendicular patterned media," Appl. Phys. Lett. 90, 162516 (2007).

${ }^{3}$ N. Eibagi, J. J. Kan, F. E. Spada, and E. E. Fullerton, "Role of Dipolar Interactions on the Thermal Stability of High-Density Bit-Patterned Media," IEEE Magn. Lett. 3, 4500204 (2012).

${ }^{4}$ B. Pfau, C. M. G. E. Guehrs, T. Hauet, T. Hennen, S. Eisebitt, and O. Hellwig, "Influence of stray fields on the switching-field distribution for bit-patterned media based on pre-patterned substrates," Appl. Phys. Lett. 105, 132407 (2014).

${ }^{5}$ T. Thomson, G. Hu, and B. D. Terris, "Intrinsic Distrib. of Magnetic Anisotropy in Thin Films Probed by Patterned Nanostructures," Phys. Rev. Lett. 96, 257204 (2006).

${ }^{6}$ J. W. Lau, R. D. McMichael, S. H. Chung, J. O. Rantschler, V. Parekh, and D. Litvinov, "Microstructural origin of switching field distribution in patterned Co/Pd multilayer nanodots," Appl. Phys. Lett. 92, 012506 (2008).

${ }^{7}$ B. Pfau, C. M. Günther, E. Guehrs, T. Hauet, H. Yang, L. Vinh, X. Xu, D. Yaney, R. Rick, S. Eisebitt, and O. Hellwig, "Origin of magnetic switching field distribution in bit patterned media based on pre-patterned substrates," Appl. Phys. Lett. 99, 062502 (2011).

${ }^{8}$ Y. Chen, J. Ding, J. Deng, T. Huang, S. H. Leong, J. Shi, B. Zong, H. Y. Y. Ko, C. K. Au, S. Hu, and B. Liu, "Switching Probability Distrib. of Bit Islands in Bit Patterned Media," IEEE Trans. Magn. 46, 1990 (2010).

${ }^{9}$ W. M. Li, Y. J. Chen, T. L. Huang, J. M. Xue, and J. Ding, "Calculation of individual bit island switching field distribution in perpendicular magnetic bit patterned media," J. Appl. Phys. 109, 07 B758 (2011).

${ }^{10}$ O. Hellwig, J. K. Bosworth, E. Dobisz, D. Kercher, T. Hauet, G. Zeltzer, J. D. Risner-Jamtgaard, D. Yaney, and R. Ruiz, "Bit patterned media based on block copolymer directed assembly with narrow magnetic switching field distribution," Appl. Phys. Lett. 96, 052511 (2010).

${ }^{11}$ F. Büttner, C. Moutafis, A. Bisig, P. Wohlhüter, C. M. Günther, J. Mohanty, J. Geilhufe, M. Schneider, C. V. Korff Schmising, S. Schaffert, B. Pfau, M. Hantschmann, M. Riemeier, M. Emmel, S. Finizio, G. Jakob, M. Weigand, J. Rhensius, J. H. Franken, R. Lavrijsen, H. J. M. Swagten, H. Stoll, S. Eisebitt, and M. Kläui, "Magnetic states in low-pinning highanisotropy material nanostructures suitable for dynamic imaging," Phys. Rev. B 87, 134422 (2013).

${ }^{12}$ S. Eisebitt, J. Lüning, W. F. Schlotter, M. Lörgen, O. Hellwig, W. Eberhardt, and J. Stöhr, "Lensless imaging of magnetic nanostructures by X-ray spectro-holography," Nature 432, 885 (2004).

${ }^{13}$ B. Pfau and S. Eisebitt, "X-ray holography," in Synchrotron Light Sources and Free-Electron Lasers, edited by E. Jaeschke, S. Khan, J. Schneider, and J. Hastings (Springer International Publishing, 2016), pp. 1093-1133. 
${ }^{14}$ M. P. Sharrock, "Time dependence of switching fields in magnetic recording media (invited)," J. Appl. Phys. 76, 6413-6418 (1994).

${ }^{15}$ C. P. Bean and J. D. Livingston, "Superparamegnetism," J. Appl. Phys. 30, S120 (1959)

${ }^{16}$ P. Krone, D. Makarov, M. Albrecht, T. Schrefl, and D. Suess, "Magnetization reversal processes of single nanomagnets and their energy barrier,” J. Magn. Magn. Mater. 322, 3771 (2010).

${ }^{17}$ J. B. C. Engelen, M. Delalande, A. J. le Fèbre, T. Bolhuis, T. Shimatsu, N. Kikuchi, L. Abelmann, and J. C. Lodder, "Thermally induced switching field distribution of a single CoPt dot in a large array," Nanotechnol. 21, 035703 (2010).

${ }^{18}$ R. Skomski, J. Zhou, R. D. Kirby, and D. J. Sellmyer, "Micromagnetic energy barriers," J. Appl. Phys. 99, 08 B906 (2006).

${ }^{19}$ J. Kurkilärvi, "Intrinsic Fluctuations in a Superconducting Ring Closed with a Josephson Junction," Phys. Rev. B 6, 832 (1972).

${ }^{20}$ A. Garg, "Escape-field distribution for escape from a metastable potential well subject to a steadily increasing bias field," Phys. Rev. B 51, 15592 (1995).

${ }^{21}$ L. Gunther and B. Barbara, "Quantum tunneling across a domain-wall junction,” Phys. Rev. B 49, 3926 (1994).
${ }^{22}$ W. Wernsdorfer, E. Orozco, and K. Hasselbach, "Experimental evidence of the Néel-Brown model of magnetization reversal," Phys. Rev. Lett. 78, 1791 (1997).

${ }^{23}$ H.-T. Wang, S. T. Chui, A. Oriade, and J. Shi, "Temperature dependence of the fluctuation of the switching field in small magnetic structures," Phys. Rev. B 69, 064417 (2004).

${ }^{24}$ B. N. Engel, C. D. England, R. A. Van Leeuwen, M. H. Wiedmann, and C. M. Falco, "Interface magnetic anisotropy in epitaxial superlattices," Phys. Rev. Lett. 67, 1910 (1991).

${ }^{25}$ I. Tudosa, M. V. Lubarda, K. T. Chan, M. A. Escobar, V. Lomakin, and E. E. Fullerton, "Qualitative insight and quantitative analysis of the effect of temperature on the coercivity of a magnetic system," Appl. Phys. Lett. 100, 102401 (2012).

${ }^{26}$ L. Breth, D. Suess, C. Vogler, B. Bergmair, M. Fuger, R. Heer, and H. Brueck, "Thermal switching field distribution of a single domain particle for field-dependent attempt frequency," J. Appl. Phys. 112, 023903 (2012).

${ }^{27}$ O. Hovorka, J. Pressesky, G. Ju, A. Berger, and R. W. Chantrell, "Ratedependence of the switching field distribution in nanoscale granular magnetic materials,” Appl. Phys. Lett. 101, 182405 (2012). 